\title{
MINIMAL SUBMANIFOLDS OF A SPHERE WITH BOUNDED SECOND FUNDAMENTAL FORM
}

\author{
HILLEL GAUCHMAN
}

\begin{abstract}
Let $h$ be the second fundamental form of an $n$-dimensional minimal submanifold $M$ of a unit sphere $S^{n+p}(p \geq 2), S$ be the square of the length of $h$, and $\sigma(u)=\|h(u, u)\|^{2}$ for any unit vector $u \in T M$. Simons proved that if $S \leq n /(2-1 / p)$ on $M$, then either $S \equiv 0$, or $S \equiv n /(2-1 / p)$. Chern, do Carmo, and Kobayashi determined all minimal submanifolds satisfying $S \equiv n /(2-1 / p)$. In this paper the analogous results for $\sigma(u)$ are obtained. It is proved that if $\sigma(u) \leq \frac{1}{3}$, then either $\sigma(u) \equiv 0$, or $\sigma(u) \equiv \frac{1}{3}$. All minimal submanifolds satisfying $\sigma(u)$ are determined. A stronger result is obtained if $M$ is odd-dimensional.
\end{abstract}

1. Introduction. Let $M$ be a smooth (i.e. $C^{\infty}$ ) compact $n$-dimensional Riemannian manifold minimally immersed in a unit sphere $S^{n+p}$ of dimension $n+p$. Let $h$ be the second fundamental form of the immersion. $h$ is a symmetric bilinear mapping $T_{x} \times T_{x} \rightarrow T_{x}^{\perp}$ for $x \in M$, where $T_{x}$ is the tangent space of $M$ at $x$ and $T_{x}^{\perp}$ is the normal space to $M$ at $x$. We denote by $S(x)$ the square of the length of $h$ at $x$. By the equation of Gauss, $S(x)=n(n-1)-\rho(x)$, where $\rho(x)$ is the scalar curvature of $M$ at $x$. Therefore, $S(x)$ is an intrinsic invariant of $M$. Let $\Pi: U M \rightarrow M$ and $U M_{x}$ be the unit tangent bundle of $M$ and its fiber over $x \in M$, respectively. We set $\sigma(u)=\|h(u, u)\|^{2}$ for any $u$ in $U M . \sigma(u)$ is not an intrinsic invariant of $M$. However, like $S(x), \sigma(u)$ is a measure of an immersion from being totally geodesic.

J. Simons in [6] proved that if $S(x) \leq n /(2-1 / p)$ everywhere on $M$, then either $S(x) \equiv 0$ (i.e. $M$ is totally geodesic), or $S(x) \equiv n /(2-1 / p)$. In [1] , S.-S. Chern, M. do Carmo, and S. Kobayashi determined all minimal submanifolds $M$ of $S^{n+p}$ satisfying $S(x) \equiv n /(2-1 / p)$ (for $p=1$ it was also obtained by B. Lawson [2]). The purpose of the present paper is to obtain the analogous results for $\sigma(u)$.

To present our results we first describe the following examples of minimal immersions $[\mathbf{1}, \mathbf{5}]$.

A. Let $S^{m}(r)$ be an $m$-dimensional sphere in $\mathbf{R}^{m+1}$ of radius $r$. We imbed $S^{m}\left(\sqrt{\frac{1}{2}}\right) \times S^{m}\left(\sqrt{\frac{1}{2}}\right)$ into $S^{2 m+1}=S^{2 m+1}(1)$ as follows. Let $\xi, \eta \in S^{m}\left(\sqrt{\frac{1}{2}}\right)$. Then $\xi$ and $\eta$ are vectors in $\mathbf{R}^{m+1}$ of length $\sqrt{\frac{1}{2}}$. We can consider $(\xi, \eta)$ as a unit vector in $\mathbf{R}^{2 m+2}=\mathbf{R}^{m+1} \times \mathbf{R}^{m+1}$. It is easy to see that $S^{m}\left(\sqrt{\frac{1}{2}}\right) \times S^{m}\left(\sqrt{\frac{1}{2}}\right)$ is a minimal submanifold of $S^{2 m+1}$.

Received by the editors January 24, 1986

1980 Mathematics Subject Classification (1985 Revision). Primary 53C42. 
B. Let $F$ be the field $\mathbf{R}$ of real numbers, the field $\mathbf{C}$ of complex numbers, or the field $Q$ of quaternions. Define $d$ by

$$
d= \begin{cases}1, & \text { if } F=\mathbf{R}, \\ 2, & \text { if } F=\mathbf{C}, \\ 4, & \text { if } F=Q .\end{cases}
$$

Let $F P^{2}$ denote the projective plane over $F . F P^{2}$ is considered as the quotient space of the unit $(3 d-1)$-dimensional sphere $S^{3 d-1}(1)=\left\{x \in F^{3}:{ }^{t} \bar{x} \cdot x=1\right\}$ obtained by identifying $x$ with $\lambda x$ where $\lambda \in F$ such that $|\lambda|=1$. The canonical metric $g_{0}$ in $F P^{2}$ is the invariant metric such that the fibering $\pi: S^{3 d-1}(1) \rightarrow F P^{2}$ is a Riemannian submersion. The sectional curvature of $\mathbf{R} P^{2}$ is 1 , the holomorphic sectional curvature of $\mathbf{C} P^{2}$ is 4 , and the $Q$-sectional curvature of $Q P^{2}$ is 4 , with respect to the metric $g_{0}$. Let $\mathcal{M}(3, F)$ be the vector space of all $3 \times 3$ matrices over $F$ and let

$$
\mathcal{H}(3, F)=\left\{A \in \mathcal{M}(3, F): A^{*}=A, \text { trace } A=0\right\}
$$

where $A^{*}={ }^{t} \bar{A} . \mathcal{H}(3, F)$ is a subspace of $\mathcal{M}(3, F)$ of real dimension $3 d+2$. We define the inner product in $\mathcal{H}(3, F)=\mathbf{R}^{3 d+2}$ by $\langle A, B\rangle=\frac{1}{2} \operatorname{trace}(A B)$ for $A, B \in \mathcal{H}(3, F)$. Define a map $\bar{\psi}: S^{3 d-1} \rightarrow \mathbf{R}^{3 d+2}=\mathcal{H}(3, F)$ as folows.

$$
\bar{\psi}(x)=\left[\begin{array}{ccc}
\left|x_{1}\right|^{2}-\frac{1}{3} & x_{1} \bar{x}_{2} & x_{1} \bar{x}_{3} \\
x_{2} \bar{x}_{1} & \left|x_{2}\right|^{2}-\frac{1}{3} & x_{2} \bar{x}_{3} \\
x_{3} \bar{x}_{1} & x_{3} \bar{x}_{2} & \left|x_{3}\right|^{2}-\frac{1}{3}
\end{array}\right]
$$

for $x=\left(x_{1}, x_{2}, x_{3}\right) \in S^{3 d-1}(1) \subset F^{3}$. Then, it is easily verified that $\bar{\psi}$ induces a map $\psi: F P^{2} \rightarrow \mathbf{R}^{3 d+2}=\mathcal{H}(3, F)$ such that $\bar{\psi}=\psi \circ \pi$. Direct computation shows that $\psi\left(F P^{2}\right) \subset S^{3 d+1}(1 / 3)$. We blow up the metric $g_{0}$ by putting $g=3 g_{0}$ in $F P^{2}$, so that the sectional curvature of $\mathbf{R} P^{2}$ is $\frac{1}{3}$ and the holomorphic sectional curvature (resp. $Q$-sectional curvature) of $\mathbf{C} P^{2}$ (resp. $Q P^{2}$ ) is $\frac{4}{3}$, with respect to the metric $g$. Then $\psi$ gives a map $\psi: F P^{2} \rightarrow S^{3 d+1}(1)$. It is proved in [5] that $\psi$ is an isometric minimal imbedding. Thus, we have the following isometric minimal imbeddings:

$$
\begin{aligned}
& \psi_{1}: \mathbf{R} P^{2} \rightarrow S^{4}(1) \quad(\text { the Veronese surface), } \\
& \psi_{2}: \mathbf{C} P^{2} \rightarrow S^{7}(1), \\
& \psi_{3}: Q P^{2} \rightarrow S^{13}(1) .
\end{aligned}
$$

In a similar manner one may obtain (see [5] for details) an isometric imbedding of the Cayley projective plane Cay $P^{2}$ furnished with the canonical metric (normalized such that the $C$-sectional curvature equals $\left.\frac{4}{3}\right)$ into $S^{25}(1)$ :

$$
\psi_{4} \text { : Cay } P^{2} \rightarrow S^{25}(1) \text {. }
$$

In addition there is an immersion

$$
\psi_{1}^{\prime}: S^{2}(\sqrt{3}) \rightarrow S^{4}(1)
$$

defined by $\psi_{1}^{\prime}=\psi_{1} \circ \pi$.

For $n, m \geq 0$, let $S^{n}(1)$ be the great sphere in $S^{n+m}(1)$ given by

$$
S^{n}(1)=\left\{\left(x_{1}, \ldots, x_{n+m+1}\right) \in S^{n+m}(1): x_{n+2}=\cdots=x_{n+m+1}=0\right\},
$$


and $\tau_{n, m}: S^{n}(1) \rightarrow S^{n+m}(1)$ be the inclusion. For $p=0,1, \ldots$, we set

$$
\begin{aligned}
& \phi_{1, p}=\tau_{4, p} \circ \psi_{1}: \mathbf{R} P^{2} \rightarrow S^{4+p}, \\
& \phi_{2, p}=\tau_{7, p} \circ \psi_{2}: \mathbf{C} P^{2} \rightarrow S^{7+p}, \\
& \phi_{3, p}=\tau_{13, p} \circ \psi_{3}: Q P^{2} \rightarrow S^{13+p}, \\
& \phi_{4, p}=\tau_{25, p} \circ \psi_{4}: \text { Cay } P^{2} \rightarrow S^{25+p}, \\
& \phi_{1, p}^{\prime}=\tau_{4, p} \circ \psi_{1}^{\prime}: S^{2}(\sqrt{3}) \rightarrow S^{4+p} .
\end{aligned}
$$

$\phi_{i, p}(i=1, \ldots, 4 ; p=0,1, \ldots)$, is an isometric minimal imbedding and $\phi_{1, p}^{\prime}(p=$ $0,1, \ldots)$, is an isometric minimal immersion.

We now state the results of the present paper.

THEOREM 1. Let $M$ be a compact $n$-dimensional manifold minimally immersed in a unit sphere $S^{n+1}$. Assume that $n(=2 m)$ is even.

(i) If $\sigma(u)<1$ for any $u \in U M$, then $M$ is totally geodesic in $S^{n+1}$.

(ii) If $\max _{u \in U M} \sigma(u)=1$, then $M$ is $S^{m}\left(\frac{1}{2}\right) \times S^{m}\left(\frac{1}{2}\right)$ minimally imbedded in $S^{2 m+1}$ as described above.

THEOREM 2. Let $M$ be a compact $n$-dimensional manifold minimally immersed in a unit sphere $S^{n+1}$. Assume that $n(=2 m+1)$ is odd. If $\sigma(u) \leq 1 /(1-1 / n)$ for any $u \in U M$, then $M$ is totally geodesic in $S^{n+1}$.

REMARK. Theorems $1(\mathrm{i})$ and 2 are easy consequences of J. Simons' results [6]. The only nontrivial part of Theorem 1 (ii) is that $\max _{u \in U M} \sigma(u)=1$ implies $S(x) \equiv n$ on $U M$. The remaining part of Theorem 1 (ii) readily follows from results of S.-S. Chern, M. do Carmo, S. Kobayashi [1], and B. Lawson [2]. We present Theorems 1 and 2 mainly for completeness. Our main results are Theorems 3 and 4.

THEOREM 3. Let $M$ be a compact $n$-dimensional manifold minimally immersed in a unit sphere $S^{n+p}$. Assume that $p \geq 2$ and $n(=2 m)$ is even.

(i) If $\sigma(u)<\frac{1}{3}$ for any $u \in U M$, then $M$ is totally geodesic in $S^{n+p}$.

(ii) If $\max _{u \in U M} \sigma(u)=\frac{1}{3}$, then $\sigma(u) \equiv \frac{1}{3}$ on $U M$, and the immersion of $M$ into $S^{n+p}$ is one of the imbeddings $\phi_{i, p}(i=1, \ldots, 4 ; p=0,1, \ldots)$, or the immersions $\phi_{1, p}^{\prime}(p=0,1, \ldots)$, described above.

THEOREM 4. Let $M$ be a compact $n$-dimensional manifold minimally immersed in a unit sphere $S^{n+p}$. Asume that $p \geq 2$ and $n(=2 m+1)$ is odd. If $\sigma(u) \leq$ $1 /(3-2 / n)$ for any $u \in U M$, then $M$ is totally geodesic in $S^{n+p}$.

It is my pleasure to thank Samuel I. Goldberg and Gabor Toth for many helpful discussions.

2. Maximal directions. Let $M$ be a compact $n$-dimensional manifold minimally immersed in $S^{n+p}$. We choose a local field of adapted orthonormal frames in $S^{n+p}$, that is frames $\left\{e_{1}, \ldots, e_{n+p}\right\}$ such that the vectors $e_{1}, \ldots, e_{n}$ are tangent to $M$. The vectors $e_{n+1}, \ldots, e_{n+p}$ are therefore normal to $M$. From now on let the indices $a, b, c, \ldots$, run from $1, \ldots, n$, and the indices $\alpha, \beta, \gamma, \ldots$, run from $n+1, \ldots, n+p$. Let $h=\left(h_{a b}^{\alpha}\right)$ be the second fundamental form of the immersed 
manifold $M$, and $\sigma(u)=\|h(u, u)\|^{2}$ for $u \in U M$. Since the immersion of $M$ into $S^{n+p}$ is minimal, $\sum_{a} h_{a a}^{\alpha}=0$ for all $\alpha$.

Let $x \in M$. Suppose that $u \in U M_{x}$ satisfies $\sigma(u)=\max _{v \in U M_{x}} \sigma(v)$. We shall call $u$ a maximal direction at $x$. Let $\left\{e_{1}, \ldots, e_{n+p}\right\}$ be an adapted frame at $x$. Assume that $e_{1}$ is a maximal direction at $x, \sigma\left(e_{1}\right) \neq 0$, and $e_{n+1}=$ $h\left(e_{1}, e_{1}\right) /\left\|h\left(e_{1}, e_{1}\right)\right\|$. Because of our choice of $e_{n+1}$,

$$
h_{11}^{\alpha}=0, \quad \alpha \neq n+1 .
$$

Since $e_{1}$ is a maximal direction, we have at the point $x$ for any $t, x^{2}, \ldots, x^{n} \in \mathbf{R}$

$$
\left\|h\left(e_{1}+t \sum_{a=2}^{n} x^{a} e_{a}, e_{1}+t \sum_{a=2}^{n} x^{a} e_{a}\right)\right\|^{2} \leq\left[1+t^{2} \sum_{a=2}^{n}\left(x^{a}\right)^{2}\right]^{2}\left(h_{11}^{n+1}\right)^{2} .
$$

Expanding in terms of $t$, we obtain

$$
4 t h_{11}^{n+1} \sum_{a \neq 1} x^{a} h_{1 a}^{n+1}+O\left(t^{2}\right) \leq 0 .
$$

It follows that

$$
h_{1 a}^{n+1}=0, \quad a=2, \ldots, n .
$$

We now choose an adapted frame at $x \in M$ such that in addition to (2.1) and (2.3),

$$
h_{a b}^{n+1}=0, \quad a \neq b .
$$

Once more expanding (2.2) in terms of $t$, we obtain

$$
\begin{gathered}
-2 t^{2}\left\{\sum_{a \neq 1}\left[h_{11}^{n+1}\left(h_{11}^{n+1}-h_{a a}^{n+1}\right)-2 \sum_{\alpha \neq n+1}\left(h_{1 a}^{\alpha}\right)^{2}\right]\left(x^{a}\right)^{2}\right. \\
\left.-4 \sum_{\alpha \neq n+1} \sum_{\substack{a, b \neq 1 \\
a \neq b}} h_{1 a} h_{1 b} x^{a} x^{b}\right\}+O\left(t^{3}\right) \leq 0 .
\end{gathered}
$$

It follows that

$$
2 \sum_{\alpha \neq n+1}\left(h_{1 a}^{\alpha}\right)^{2} \leq h_{11}^{n+1}\left(h_{11}^{n+1}-h_{a a}^{n+1}\right), \quad a=2, \ldots, n .
$$

Let us define a tensor field $H=\left(H_{a b c d}\right)$ on $M$ by the formula

$$
H_{a b c d}=\sum_{\alpha} h_{a b}^{\alpha} h_{c d}^{\alpha} .
$$

It is clear that $\sigma(u)=H(u, u, u, u)$.

Lemma 1. Let $u$ be a maximal direction at $x \in M$. Assume that $\sigma(u) \neq 0$. Let $e_{1}, \ldots, e_{n+p}$ be an adapted frame at $x$ such that $e_{1}=u, e_{n+1}=h\left(e_{1}, e_{1}\right) /\left\|h\left(e_{1}, e_{1}\right)\right\|$, and $h_{a b}^{n+1}=0$ for $a \neq b$. At the point $x$

(i) if $p=1$, then

$$
\frac{1}{2}(\Delta H)_{1111} \geq\left(h_{11}^{n+1}\right)^{2}\left[n-\sum_{a}\left(h_{a a}^{n+1}\right)^{2}\right] .
$$


(ii) if $p \geq 2$, then

$$
\frac{1}{2}(\Delta H)_{1111} \geq\left(h_{11}^{n+1}\right)^{2}\left[n-n\left(h_{11}^{n+1}\right)^{2}-2 \sum_{a}\left(h_{a a}^{n+1}\right)^{2}\right]
$$

with equality attained if and only if

$$
\left(h_{11}^{n+1}-h_{a a}^{n+1}\right)\left[h_{11}^{n+1}\left(h_{11}^{n+1}-h_{a a}^{n+1}\right)-2 \sum_{\alpha \neq n+1}\left(h_{1 a}^{\alpha}\right)^{2}\right]=0
$$

and

$$
\nabla_{a} h_{11}^{\alpha}=0
$$

for all $a$ and all $\alpha$, where $\Delta$ and $\nabla_{a}$ denote the Laplacian and the covariant derivative, respectively.

PROOF.

$$
\frac{1}{2}(\Delta H)_{1111}=h_{11}^{n+1}(\Delta h)_{11}^{n+1}+\sum_{a, \alpha}\left(\nabla_{a} h_{11}^{\alpha}\right)^{2} .
$$

Using Simons' formula [6] for the Laplacian of the second fundamental form (see also [1]), we obtain

$$
\frac{1}{2}(\Delta H)_{1111}=\left(h_{11}^{n+1}\right)^{2}\left[n-\sum_{a}\left(h_{a a}^{n+1}\right)^{2}\right]+\sum_{a, \alpha}\left(\nabla_{a} h_{11}^{\alpha}\right)^{2}, \quad \text { if } p=1,
$$

and

$$
\begin{aligned}
\frac{1}{2}(\Delta H)_{1111} & =\left(h_{11}^{n+1}\right)^{2}\left[n-n\left(h_{11}^{n+1}\right)^{2}-2 \sum_{a}\left(h_{a a}^{n+1}\right)^{2}\right] \\
& +\sum_{a} h_{11}^{n+1}\left(h_{11}^{n+1}-h_{a a}^{n+1}\right)\left[h_{11}^{n+1}\left(h_{11}^{n+1}-h_{a a}^{n+1}\right)-2 \sum_{\alpha \neq n+1}\left(h_{1 a}^{\alpha}\right)^{2}\right] \\
& +\sum_{a, \alpha}\left(\nabla_{a} h_{11}^{\alpha}\right)^{2}, \quad \text { if } p \geq 2,
\end{aligned}
$$

from which the lemma follows readily by inequality (2.6).

LEMMA 2. Let an adapted frame $\left\{e_{1}, \ldots, e_{n+p}\right\}$ at $x \in M$ be as in Lemma 1.

(i) Assume that $n(=2 m)$ is even. If

$$
\sigma(u) \leq\left\{\begin{array}{ll}
1, & \text { if } p=1, \\
\frac{1}{3}, & \text { if } p \geq 2,
\end{array} \text { for all } u \in U M_{x}\right.
$$

then $(\Delta H)_{1111} \geq 0$. If equality $(\Delta H)_{1111}=0$ is attained, then it is possible to renumber $e_{1}, \ldots, e_{2 m}$ such that the following equalities hold

$$
h_{11}^{n+1}=\cdots=h_{m m}^{n+1}=-h_{m+1}^{n+1} m+1=\cdots=-h_{2 m 2 m}^{n+1}= \begin{cases}1, & \text { if } p=1, \\ 1 / \sqrt{3}, & \text { if } p \geq 2 .\end{cases}
$$


(ii) Assume that $n(=2 m+1)$ is odd. If

$$
\sigma(u) \leq\left\{\begin{array}{ll}
1-\frac{1}{n}, & \text { if } p=1, \\
\frac{1}{3-2 / n}, & \text { if } p \geq 2,
\end{array} \quad \text { for all } u \in U M_{x}\right.
$$

then $(\Delta H)_{1111} \geq 0$. If equality $(\Delta H)_{1111}=0$ is attained, then it is possible to renumber $e_{1}, \ldots, e_{2 m+1}$ such that the following equalities hold.

$$
\begin{aligned}
& h_{11}^{n+1}=\cdots=h_{m m}^{n+1}=-h_{m+1}^{n+1} m+1=\cdots=-h_{2 m 2 m}^{n+1} \\
& = \begin{cases}\left(1-\frac{1}{n}\right)^{-1 / 2}, & \text { if } p=1, \\
\left(3-\frac{2}{n}\right)^{-1 / 2}, & \text { if } p \geq 2,\end{cases} \\
& h_{2 m+12 m+1}^{n+1}=0 .
\end{aligned}
$$

PROOF. Since $e_{1}$ is a maximal direction

$$
-h_{11}^{n+1} \leq h_{a a}^{n+1} \leq h_{11}^{n+1}, \quad a=2, \ldots, n .
$$

Because of minimality of the immersion of $M$ into $S^{n+p}$,

$$
\sum_{a=2}^{n} h_{a a}^{n+1}=-h_{11}^{n+1} .
$$

It is easily seen that the convex function $f\left(h_{22}^{n+1}, \ldots, h_{n n}^{n+1}\right)=\sum_{a=2}^{n}\left(h_{a a}^{n+1}\right)^{2}$ of $(n-1)$ variables $h_{22}^{n+1}, \ldots, h_{n n}^{n+1}$ subject to the linear constraints $(2.16),(2.17)$ attains its maximal value when (after suitable renumbering of $e_{1}, \ldots, e_{n}$ )

$$
h_{11}^{n+1}=\cdots=h_{m m}^{n+1}=-h_{m+1}^{n+1}{ }_{m+1}=\cdots=-h_{2 m 2 m}^{n+1}, \quad \text { if } n=2 m,
$$

and

$$
\begin{aligned}
h_{11}^{n+1}=\cdots=h_{m m}^{n+1}=-h_{m+1}^{n+1} m+1 & =\cdots= \\
& -h_{2 m 2 m}^{n+1}, \\
& h_{2 m+12 m+1}^{n+1}=0, \quad \text { if } n=2 m+1 .
\end{aligned}
$$

Therefore, by inequalities $(2.8),(2.9)$,

$$
\frac{1}{2}(\Delta H)_{1111} \geq \begin{cases}n\left(h_{11}^{n+1}\right)^{2}\left[1-\sigma\left(e_{1}\right)\right], & \text { if } p=1, n=2 m, \\ n\left(h_{11}^{n+1}\right)^{2}\left[1-3 \sigma\left(e_{1}\right)\right], & \text { if } p \geq 2, n=2 m, \\ \left(h_{11}^{n+1}\right)^{2}\left[n-(n-1) \sigma\left(e_{1}\right)\right], & \text { if } p=1, n=2 m+1, \\ \left(h_{11}^{n+1}\right)^{2}\left[n-(3 n-2) \sigma\left(e_{1}\right)\right], & \text { if } p \geq 2, n=2 m+1\end{cases}
$$

This proves the lemma.

Let $L(x)$ be a function on $M$ defined by $L(x)=\max _{u \in U M_{x}} \sigma(u)$.

LEMma 3. Assume that one of $A_{1}, A_{2}, A_{3}, A_{4}$ is satisfied.

$\left(\mathrm{A}_{1}\right) p=1, n$ is even, $\sigma(u) \leq 1$ for all $u \in U M$,

( $\left.\mathrm{A}_{2}\right) p=1, n$ is odd, $\sigma(u) \leq 1 /(1-1 / n)$ for all $u \in U M$,

$\left(\mathrm{A}_{3}\right) p \geq 2, n$ is even, $\sigma(u) \leq \frac{1}{3}$ for all $u \in U M$,

$\left(\mathrm{A}_{4}\right) p \geq 2, n$ is odd, $\sigma(u) \leq 1 /(3-2 / n)$ for all $u \in U M$.

Then $L(x)$ is a constant function on $M$. 
ProOF. Following an idea in $[3]$ we prove the lemma using the maximum principle. Clearly $L(x)$ is a continuous function. It suffices to show that $L(x)$ is subharmonic in the generalized sense. Fix $x \in M$ and let $e_{1}$ be a maximal direction at $x$. In an open neighborhood $U_{x}$ of $x$ within the cut-locus of $x$ we shall denote by $u(y)$ the tangent vector to $M$ obtained by parallel transport of $e_{1}=u(x)$ along the unique geodesic joining $x$ to $y$ within the cut-locus of $x$. Define $g_{x}(y)=\sigma(u(y))$. Then

$$
\begin{aligned}
\Delta g_{x}(x) & =\Delta[H(u(y), u(y), u(y), u(y))]_{y=x} \\
& =\sum_{a}\left(\nabla_{a}^{2} H\right)\left(e_{1}, e_{1}, e_{1}, e_{1}\right)=(\Delta H)_{1111}(x) .
\end{aligned}
$$

If $\left\|h\left(e_{1}, e_{1}\right)\right\| \neq 0$, then by Lemma $2,(\Delta H)_{1111}(x) \geq 0$. If $\left\|h\left(e_{1}, e_{1}\right)\right\|=0$, then $h \equiv 0$ at $x$. In this case the formula of Simons [6] for $\Delta h$ shows that $\Delta h=0$ at $x$, and therefore

$$
(\Delta H)_{1111}(x)=\sum_{a, \alpha}\left(\nabla_{a} h_{11}^{\alpha}\right)^{2} \geq 0 .
$$

Thus, we obtain that in any case $\Delta g_{x}(x)=(\Delta H)_{1111}(x) \geq 0$.

For the Laplacian of continuous functions, we have the generalized definition

$$
\Delta L=C \lim _{r \rightarrow 0} \frac{1}{r^{2}}\left(\int_{B(x, r)} L / \int_{B(x, r)} 1-L(x)\right),
$$

where $C$ is a positive constant and $B(x, r)$ denotes the geodesic ball of radius $r$ with the center at $x$. With this definition $L$ is subharmonic on $M$ if and only if $\Delta L(x) \geq 0$ at each point $x \in M$. Since $g_{x}(x)=L(x)$ and $g_{x} \leq L$ on $U_{x}, \Delta L(x) \geq \Delta g_{x}(x) \geq 0$. Thus, $L(x)$ is subharmonic and hence constant on $M$.

\section{Proofs of Theorems 1-4.}

Lemma 4. Assume that one of $B_{1}, B_{2}, B_{3}, B_{4}$ is satisfied.

$\left(\mathrm{B}_{1}\right) p=1, n$ is even, $\sigma(u)<1$ for all $u \in U M$,

$\left(\mathrm{B}_{2}\right) p=1, n$ is odd, $\sigma(u)<1 /(1-1 / n)$ for all $u \in U M$,

( $\left.\mathrm{B}_{3}\right) p \geq 2, n$ is even, $\sigma(u)<\frac{1}{3}$ for all $u \in U M$,

$\left(\mathrm{B}_{4}\right) p \geq 2, n$ is odd, $\sigma(u)<1 /(3-2 / n)$ for all $u \in U M$.

Then $M$ is totally geodesic in $S^{n+p}$.

PROOF. Let $x \in M$ and $e_{1}$ be a maximal direction at $x$. Assume that $\sigma\left(e_{1}\right) \neq 0$. Let $g_{x}(y)=\sigma(u(y))$ be the function defined in the proof of Lemma 3. By Lemma $3, g_{x}(x)$ is a maximum of $g_{x}$. Therefore, $(\Delta H)_{1111}(x)=\Delta g_{x}(x) \leq 0$. On the other hand, by Lemma $2,(\Delta H)_{1111}(x) \geq 0$. Therefore, $(\Delta H)_{1111}=0$ on $M$. Hence, by (2.14) and (2.15),

$$
\sigma\left(e_{1}\right)= \begin{cases}\frac{1,}{1-1 / n}, & \text { if } p=1, n \text { is even } \\ \frac{1}{3}, & \text { if } p \geq 2, n \text { is odd } \\ \frac{1}{3-2 / n}, & \text { if } p \geq 2, n \text { is odd }\end{cases}
$$


contradicting the assumptions $B_{1}, B_{2}, B_{3}, B_{4}$. Hence, $h(u, u)=0$ for all $u \in U M$, that is $M$ is totally geodesic in $S^{n+p}$.

PROOF OF THEOREM 1. (i) follows from Lemma 4. We prove (ii). As in the poof of Lemma 4, we obtain $(\Delta H)_{1111}=0$. Hence, by (2.4) and (2.14),

$$
S(x)=\sum_{\alpha, a, b}\left(h_{a b}^{\alpha}\right)^{2}=\sum_{a}\left(h_{a a}^{n+1}\right)^{2}=n .
$$

All minimal immersions into $S^{n+1}$ satisfying $S(x) \equiv n$ were found by S.-S. Chern, M. do Carmo, and S. Kobayashi in [1] and B. Lawson in [2]. It is easy to see that among their immersions only $S^{m}\left(\sqrt{\frac{1}{2}}\right) \times S^{m}\left(\sqrt{\frac{1}{2}}\right)$ imbedded in $S^{2 m+1}$ satisfies the condition $\max _{u \in U M} \sigma(u)=1$. This completes the proof of Theorem 1 .

PROOF OF THEOREM 2. By Lemmas 3 and 4, we have to consider only the case $L(x)=\max _{u \in U M_{x}} \sigma(u) \equiv 1 /(1-1 / n)$ on $M$. As in the proof of Lemma 4, $(\Delta H)_{1111}=0$. Hence, by $(2.15)$,

$$
S(x) \equiv \sum_{\alpha, a, b}\left(h_{a b}^{\alpha}\right)^{2} \equiv \sum_{a=1}^{n+1} \frac{1}{(1-1 / n)} \equiv n .
$$

It is shown in [1] that if $M$ is minimally immersed in $S^{n+1}$ and $S(x) \equiv n$, then $h_{a a}^{n+1}$ may attain at most two different values for $a=1, \ldots, n$. However, since by $(2.15)$,

$$
h_{11}^{n+1}=\left(\frac{n}{n-1}\right)^{1 / 2}, \quad h_{m+1 m+1}^{n+1}=-\left(\frac{n}{n-1}\right)^{1 / 2}, \quad h_{2 m+12 m+1}^{n+1}=0,
$$

we obtain a contradiction, so the equality $\max _{u \in U M} \sigma(u) \equiv 1 /(1-1 / n)$ on $U M$ is impossible. This completes the proof of Theorem 2 .

PROOF OF THEOREM 3. (i) follows from Lemma 4. We prove (ii). As in the proof of Lemma 4 , we obtain $(\Delta H)_{1111}=0$. Let the indices $i, j, k, \ldots$, run from $1, \ldots, m$, and let $\bar{i}, \bar{j}, \bar{k}, \ldots$, denote $i+m, j+m, k+m, \ldots$, respectively. By (2.14) we have

$$
h_{i i}^{n+1}=-h_{\bar{i} \bar{i}}^{n+1}=-1 / \sqrt{3}, \quad i=1, \ldots, m .
$$

Since $\left\|h\left(e_{i}, e_{i}\right)\right\|^{2} \leq \frac{1}{3}$ and $\left\|h\left(e_{\bar{i}}, e_{\bar{i}}\right)\right\|^{2} \leq \frac{1}{3}$, we obtain

$$
h_{i i}^{\alpha}=h_{i \bar{i}}^{\alpha}=0, \quad \alpha \neq n+1 ; i=1, \ldots, m .
$$

By (2.10), $\sum_{\alpha \neq n+1}\left(h_{1 \bar{j}}^{\alpha}\right)^{2}=\frac{1}{3}$. Since each vector $e_{a},(a=1, \ldots, n)$, is a maximal direction,

$$
\sum_{\alpha \neq n+1}\left(h_{i j}^{\alpha}\right)^{2}=\frac{1}{3}, \quad i, j=1, \ldots, m .
$$

Let $u=\left(e_{i}+e_{j}\right) / \sqrt{2}$. Then

$$
\begin{aligned}
\sigma(u) & =\frac{1}{4}\left\|h\left(e_{i}+e_{j}, e_{i}+e_{j}\right)\right\|^{2} \\
& =\frac{1}{4}\left\|\left(h_{i i}^{n+1}+h_{j j}^{n+1}\right) e_{n+1}+2 \sum_{\alpha \neq n+1} h_{i j}^{\alpha} e_{\alpha}\right\|^{2} \\
& =\frac{1}{3}+\sum_{\alpha \neq n+1}\left(h_{i j}^{\alpha}\right)^{2} \leq \frac{1}{3} .
\end{aligned}
$$


Therefore,

$$
h_{i j}^{\alpha}=0, \quad \alpha \neq n+1 ; i, j=1, \ldots, m
$$

Similarly,

$$
h_{i \bar{j}}^{\alpha}=0, \quad \alpha \neq n+1 ; i, j=1, \ldots, m .
$$

Expansion (2.5) now takes the form

$$
t^{2}\left(-4 \sum_{\alpha} \sum_{j \neq k} h_{i \bar{j}}^{\alpha} h_{i \bar{k}}^{\alpha} x^{\bar{j}} x^{\bar{k}}\right)+O\left(t^{3}\right) \leq 0 .
$$

It follows that $\sum_{\alpha} h_{1 \bar{j}}^{\alpha} h_{1 \bar{k}}^{\alpha}=0$ for $j \neq k$. Since each vector $e_{a}$ is a maximal direction,

$$
\begin{array}{ll}
\sum_{\alpha} h_{i \bar{j}}^{\alpha} h_{i \bar{k}}^{\alpha}=0, & j \neq k, \\
\sum_{\alpha} h_{i \bar{k}}^{\alpha} h_{j \bar{k}}^{\alpha}=0, & i \neq j .
\end{array}
$$

Once more expanding (2.2) in terms of $t$,

$$
2 t^{3} \sum_{\alpha, j, k, l}\left(h_{1 \bar{k}}^{\alpha} h_{j \bar{l}}^{\alpha}+h_{1 \bar{l}}^{\alpha} h_{j \bar{k}}^{\alpha}\right) x^{j} x^{\bar{k}} x^{\bar{l}}+O\left(t^{4}\right) \leq 0,
$$

from which

$$
\sum_{\alpha}\left(h_{i \bar{k}}^{\alpha} h_{j \bar{l}}^{\alpha}+h_{i \bar{l}}^{\alpha} h_{j \bar{k}}^{\alpha}\right)=0, \quad i \neq j \text { or } k \neq l .
$$

Using (2.4) and (3.1)-(3.8), we obtain by direct computation that $\sigma(u)=\frac{1}{3}$ for any $u \in U M$. B. O'Neill [4] calls an immersion $\lambda$-isotropic if $\|h(u, u)\|=\lambda$ for any $u \in U M$. Therefore, the immersion under consideration is $1 / \sqrt{3}$-isotropic.

By Lemma $1, \nabla_{a} h_{11}^{\alpha}=0$. It follows that $\nabla_{a} h_{b b}^{\alpha}=0$. By polarization, $\nabla_{a} h_{b c}^{\alpha}=$ 0 for all $\alpha, a, b, c$. Therefore, the second fundamental form of the immersion is parallel. All $\lambda$-isotropic minimal immersions into a unit sphere with parallel second fundamental form were completely classified by K. Sakamoto in [5]. Among his immersions only $\phi_{1, p}, \phi_{2, p}, \phi_{3, p}, \phi_{4, p}$ and $\phi_{1, p}^{\prime}$ described in $\S 1$, are $1 / \sqrt{3}$-isotropic. This completes the proof of the theorem.

PROOF OF THEOREM 4. By Lemmas 3 and 4, we need only consider the case $L(x) \equiv 1 /(3-2 / n)$ on $M$. We show that this case cannot occur. Thus, assume that $L(x) \equiv 1 /(3-2 / n)$ on $M$. As in the proof of Lemma $4,(\Delta H)_{1111}=0$. Let the indices $i, j, k, \ldots$, run from $1, \ldots, m$, and let $\bar{i}, \bar{j}, \bar{k}, \ldots$, denote $i+m, j+m$, $k+m, \ldots$, respectively. By $(2.15)$,

$$
\begin{aligned}
& h_{i i}^{n+1}=-h_{i \bar{i}}^{n+1}=(3-2 / n)^{1 / 2}, \quad i=1, \ldots, m, \\
& h_{n n}^{n+1}=0 .
\end{aligned}
$$

As in the proof of Theorem 3,

$$
h_{i j}^{\alpha}=h_{i \bar{j}}^{\alpha}=0, \quad \alpha \neq n+1 ; i, j=1, \ldots, m .
$$


Since $h_{n n}^{\alpha}=-\sum_{i} h_{i i}^{\alpha}-\sum_{i} h_{\bar{i} \bar{i}}^{\alpha}$,

$$
h_{n n}^{\alpha}=0 .
$$

By (2.10),

$$
\begin{aligned}
& \sum_{\alpha}\left(h_{i \bar{j}}^{\alpha}\right)^{2}=\frac{1}{3-2 / n}, \quad i, j=1, \ldots, m \\
& \sum_{\alpha}\left(h_{i n}^{\alpha}\right)^{2}=\frac{1}{2(3-2 / n)}, \quad i=1, \ldots, m, \\
& \sum_{\alpha}\left(h_{i n}^{\alpha}\right)^{2}=\frac{1}{2(3-2 / n)}, \quad i=1, \ldots, m .
\end{aligned}
$$

As in the proof of Theorem 3, we obtain with the help of expansion (2.2) the following equalities:

$$
\begin{aligned}
& \sum_{\alpha} h_{i \bar{j}}^{\alpha} h_{i \bar{k}}^{\alpha}=0, \\
& \sum_{\alpha}^{\alpha} h_{i \bar{k}}^{\alpha} h_{j \bar{k}}^{\alpha}=0, \\
& \sum_{\alpha}^{\alpha} h_{i \bar{j}}^{\alpha} h_{i n}^{\alpha}=0, \\
& \sum_{\alpha}^{\alpha} h_{i \bar{j}}^{\alpha} h_{n \bar{j}}^{\alpha}=0, \\
& \sum_{\alpha}^{\alpha}\left(h_{i \bar{k}}^{\alpha} h_{j \bar{l}}^{\alpha}+h_{i \bar{l}}^{\alpha} h_{j \bar{k}}^{\alpha}\right)=0, \quad i \neq j \text { or } k \neq 1, \\
& \sum_{\alpha}^{\alpha}\left(h_{i \bar{k}}^{\alpha} h_{j n}^{\alpha}+h_{j \bar{k}}^{\alpha} h_{i n}^{\alpha}\right)=0, \quad i \neq j, \\
& \sum_{\alpha}^{\alpha}\left(h_{i \bar{j}}^{\alpha} h_{n \bar{k}}^{\alpha}+h_{i \bar{k}}^{\alpha} h_{n \bar{j}}^{\alpha}\right)=0, \quad j \neq k, \\
& \sum_{\alpha}^{\alpha} h_{i n}^{\alpha} h_{j n}^{\alpha}=0, \quad i \neq j, \\
& \sum_{\alpha}^{\infty} h_{i n}^{\alpha} h_{\bar{j} n}^{\alpha}=0, \quad i \neq j, \\
& \sum_{\alpha}^{\alpha} h_{i n}^{\alpha} h_{\bar{j} n}^{\alpha}=0 .
\end{aligned}
$$

Let $u=\sum_{a} u^{a} e_{a} \in U M$. Direct computation with the help of (2.4) and (3.9)-(3.24) shows that

$$
\sigma(u)=\left[1-\left(u^{n}\right)^{4}\right](3-2 / n)^{-1} .
$$

It follows from (3.25) that for any $x \in M$, the tangent space $T_{x}$ of $M$ at $x$ is a direct sum of two mutually orthogonal subspaces $T_{x}=P_{x}+Q_{x}$, where $P_{x}$ is $2 m$-dimensional and is defined by

$$
P_{x}=\left\{X \in T_{x}:\|h(X, X)\|=(3-2 / n)^{-1 / 2}\|X\|^{2}\right\},
$$


and $Q_{x}$ is 1-dimensional and is defined by

$$
Q_{x}=\left\{X \in T_{x}: h(X, X)=0\right\}
$$

LEMMA 5. The distributions $P: x \rightarrow P_{x}$ and $Q: x \rightarrow Q_{x}$ are smooth distributions on $M$.

PROOF. It is sufficient to prove that $Q$ is smooth. Let $x_{0} \in M$ and $\left\{e_{1}, \ldots, e_{n+p}\right\}$ be a smooth local field of orthonormal adapted frames in a neighborhood $U$ of $x_{0}$ such that $e_{n}\left(x_{0}\right) \in Q_{x_{0}}$. If $U$ is sufficiently small, there is a unique vector $X$ of the form $X=\sum_{a=1}^{2 m} X^{a} e_{a}+e_{n}$ which belongs to $Q_{x}$ at each point $x \in U$. We prove that $X^{a}, a=1, \ldots, 2 m$, are smooth functions of $x$.

By (3.27), $X^{a}(x), a=1, \ldots, 2 m$, are a unique solution of the system of equations

$$
\begin{aligned}
h^{\alpha}(X, X)=\sum_{a, b=1}^{2 m} h_{a b}^{\alpha}(x) X^{a} X^{b}+2 \sum_{a=1}^{2 m} h_{a n}^{\alpha}(x) X^{a} & =0, \\
\alpha & =n+1, \ldots, n+p .
\end{aligned}
$$

At the point $x_{0}$ the Jacobian of system (3.28) is

$$
\left(\partial h^{\alpha} / \partial X^{a}\right)=2\left(h_{a n}^{\alpha}\right), \quad \alpha=n+1, \ldots, n+p ; a=1, \ldots, 2 m
$$

By (3.13), (3.14) and (3.22)-(3.24), the rows of the matrix $\left(h_{a n}^{\alpha}\right)$ are mutually orthogonal nonzero vectors. Hence, $\operatorname{rank}\left(\partial h^{\alpha} / \partial X^{a}\right)=2 m$ at $x_{0}$. Therefore, $X^{a}$, $a=1, \ldots, 2 m$, are smooth functions of $x$ in a sufficiently small neighborhood of $x_{0}$.

We now return to the proof of Theorem 4. Let $x \in M$. By Lemma 5, we may choose a smooth family of orthonormal adapted frames $\left\{e_{1}, \ldots, e_{n+p}\right\}$ in some neighborhood $U$ of $x$ such that equations (2.4), (3.9)-(3.24) are satisfied on $U$. Set

$$
N_{a}=\left[2\left(3-\frac{2}{n}\right)\right]^{1 / 2} \sum_{\alpha} h_{a n}^{\alpha} e_{\alpha}, \quad a=1, \ldots, 2 m
$$

By (2.4), (3.13), (3.14), and (3.22)--(3.24), the vectors $e_{n+1}, N_{1}, \ldots, N_{2 m}$ are orthonormal. Therefore, with no loss of generality, we may assume that $e_{n+1+a}=N_{a}$, $a=1, \ldots, 2 m$. Then,

$$
\begin{aligned}
& h_{i n}^{n+1+i}=h_{\overline{i n}}^{n+1+\bar{i}}=\left[2\left(3-\frac{2}{n}\right)\right]^{1 / 2}, \quad i=1, \ldots, m, \\
& h_{i n}^{\alpha}=0, \quad \alpha \neq n+1+i, i=1, \ldots, m, \\
& h_{\overline{i n}}^{\alpha}=0, \quad \alpha \neq n+1+\bar{i}, i=1, \ldots, m .
\end{aligned}
$$


Let the indices $A, B, C$ run from $1, \ldots, n+p$, and let $\left\{\omega^{A}\right\}$ and $\left\{\omega_{B}^{A}\right\}$ be the coframe dual to the frame $\left\{e_{A}\right\}$ and the connection forms of the Riemannian connection on $S^{n+p}$, respectively. Then,

$$
\begin{aligned}
& d \omega^{A}=\sum_{B} \omega^{B} \wedge \omega_{B}^{A}, \\
& d \omega_{B}^{A}=\sum_{C} \omega^{C} \wedge \omega_{C}^{A}+\omega^{A} \wedge \omega^{B} \\
& \omega^{\alpha}=0 \\
& \omega_{a}^{\alpha}=\sum_{b} h_{a b}^{\alpha} \omega^{b} \\
& d h_{a b}^{\alpha}-\sum_{c} h_{c b}^{\alpha} \omega_{a}^{c}-\sum_{c} h_{a c}^{\alpha} \omega_{b}^{c}+\sum_{\beta} h_{a b}^{\beta} \omega_{\beta}^{\alpha}=\sum_{c}\left(\nabla_{c} h_{a b}^{\alpha}\right) \omega^{c} .
\end{aligned}
$$

As in the proof of Theorem 3, we obtain

$$
\nabla_{c} h_{a b}^{\alpha}=0, \quad a, b=1, \ldots, 2 m ; c=1, \ldots, n .
$$

Let us take $\alpha=h+1+i, a=b=i$ in (3.36). By (2.4), (3.9)-(3.11), (3.29)-(3.31), and (3.37),

$$
-2 \sum_{k} h_{\bar{k} i}^{n+1+i} \omega_{i}^{\bar{k}}-\left[2\left(3-\frac{2}{n}\right)\right]^{-1 / 2} \omega_{i}^{n}+\left(3-\frac{2}{n}\right)^{-1 / 2} \omega_{n+1}^{n+1+i}=0 .
$$

Analogously, taking $\alpha=n+1+i, a=i, b=j \neq i$ in (3.36),

$$
-2 \sum_{k} h_{\bar{k} j}^{n+1+i} \omega_{j}^{\bar{k}}+\left(3-\frac{2}{n}\right)^{-1 / 2} \omega_{n+1}^{n+1+i}=0, \quad i \neq j .
$$

Summing (3.39) with respect to $j(j \neq i)$ and adding (3.38), we have

$$
-2 \sum_{j, k} h_{\bar{k} j}^{n+1+i} \omega_{j}^{\bar{k}}+m\left(3-\frac{2}{n}\right)^{-1 / 2} \omega_{n+1}^{n+1+i}-\left[2\left(3-\frac{2}{n}\right)\right]^{-1 / 2} \omega_{i}^{n}=0 .
$$

Let us now take $\alpha=n+1+i, a=b=\bar{k}$ in (3.36). Then,

$$
-2 \sum_{j} h_{j \bar{k}}^{n+1+i} \omega_{\bar{k}}^{j}-\left(3-\frac{2}{n}\right)^{-1 / 2} \omega_{n+1}^{n+1+i}=0 .
$$

Summing (3.41) with respect to $\bar{k}$,

$$
-2 \sum_{j, k} h_{j \bar{k}}^{n+1+i} \omega_{\bar{k}}^{j}-m\left(3-\frac{2}{n}\right)^{-1 / 2} \omega_{n+1}^{n+1+i}=0 .
$$

Finally, adding (3.40) to (3.42), we get

$$
\omega_{i}^{n}=0 \text {. }
$$

Analogously, we obtain

$$
\omega_{i}^{n}=0 .
$$


Differentiating (3.43) and using (2.4), (3.9)-(3.11), (3.29)-(3.31), and (3.4), we obtain

$$
-\sum_{\alpha, a, b} h_{i a}^{\alpha} h_{b n}^{\alpha} \omega^{a} \wedge \omega^{b}+\omega^{n} \wedge \omega^{i}=0
$$

Taking the coefficient of $\omega^{n} \wedge \omega^{i}$ in (3.45) we have $-\sum_{\alpha}\left(h_{i n}^{\alpha}\right)^{2}+1=0$. By (3.13), it gives $2(3-2 / n)=1$ and therefore $n=5 / 4$, yielding a contradiction. Therefore, the equality $\max _{u \in U M_{x}} \sigma(u) \equiv 1 /(3-2 / n)$ on $M$ is impossible. This completes the proof of Theorem 4 .

\section{REFERENCES}

1. S.-S. Chern, M. do Carmo, and S. Kobayashi, Minimal submanifolds of sphere with second fundamental form of constant length, Functional Analysis and Related Fields, SpringerVerlag, Berlin and New York, 1970, pp. 59-75.

2. B. Lawson, Local rigidity theorems for minimal hypersurfaces, Ann. of Math. (2) 89 (1969), 187-197.

3. N. Mok and J.-Q. Zhang, Curvature characterization of compact Hermitian symmetric spaces, Trans. Amer. Math. Soc. 23 (1986), 15-67.

4. B. O'Neill, Isotropic and Kähler immersions, Canad. J. Math. 17 (1965), 907-915.

5. K. Sakamoto, Planar geodesic immersions, Tôhoku Math. J. 29 (1977), 25-56.

6. J. Simons, Minimal varieties in Riemannian manifolds, Ann. of Math. (2) 88 (1968), 62-105.

Department of Mathematics, University of Illinois, 1409 W. Green Street, URBANA, ILLINOIS 61801

Department of Mathematics, Ben Gurion University of the Negev, BeerSHEVA, ISRAEL 